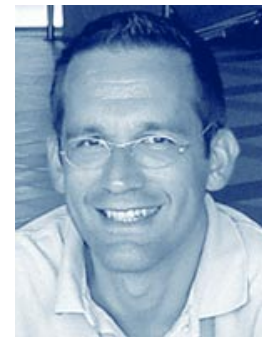

Martin E. Keck

\title{
Angsterkrankungen: Tiermodelle und Humangenetik
}

\author{
Martin E. Keck, Christoph K. Thöringer \\ Max-Planck-Institut für Psychiatrie, München
}

psychoneuro 2005; 31 (3): 139-144

A ngst zählt zu den grundlegenden Emotionen des Menschen und beeinflusst als biosoziales Signal entscheidend interpersonellen Umgang und die risikobewusste Auseinandersetzung mit der Umwelt. Angst drückt Bedrohung aus, verweist auf Gefahren und ist somit eine biologisch nicht nur sinnvolle, sondern lebenserhaltende und daher evolutionsbiologisch hochkonservierte Emotion. Durch ein Zuwenig oder ein Zuviel kann sie jedoch psychopathologische Relevanz erlangen. Angstzustände treten in diesem Kontext als Symptom psychiatrischer Erkrankungen relativ häufig auf. Angststörungen hingegen verweisen als eigene diagnostische Entität auf Ängste, deren Ausmaß und Persistenz unverhältnismäßig stark sind und mit hohem Leidensdruck sowie ausgeprägter psychosozialer Behinderung einhergehen. Aus epidemiologischer Sicht zählen Angststörungen mit einer Lebenszeitprävalenz von bis zu 25\% neben anderen affektiven Erkrankungen, wie unipolare oder bipolare Depression, zu den häufigsten Krankheitsbildern weltweit. Darüber hinaus zeigen klinische und epidemiologische Untersuchungen eine ausgeprägte Komorbidität von Angststörungen mit anderen psychiatrischen Erkrankungen wie Sucht- und affektiven Erkrankungen sowie Persönlichkeitsstörungen (8).

Nur basierend auf einem tieferen Verständnis der Neurobiologie der Angst im Tiermodell und einer kritischen Extrapolation auf den Menschen wird eine kausale Therapie von Angsterkrankungen, die zu den häufigsten psychiatrischen Krankheitsbildern gehören, möglich sein. Es gilt, jenseits der lange Zeit im Focus des Interesses gewesenen Monoamine (wie z.B. Serotonin), neurobiologische Systeme zu identifizieren, die kausal in die Ätiologie und Pathogenese der pathologischen Angst eingebunden sind. An der Ausbildung komplexer Verhaltensweisen einschließlich ihrer Erkrankungen sind viele interagierende Gene beteiligt, welche darüber hinaus in steter Wechselwirkung mit zahlreichen Umweltfaktoren stehen. Um Rückschlüsse auf die Humansituation ziehen zu können, muss sich die neurobiologische Grundlagenforschung daher auf valide Tiermodelle konzentrieren - ein Gesichtspunkt der lange Zeit vernachlässigt wurde.

\section{Tiermodelle in der psychiatrischen Grund- lagenforschung}

Zum besseren Verständnis der Regulation des physiologischen und pathologischen Angstverhaltens sowie der zugrundeliegenden neurobiologischen Mechanismen bedarf es adäquater Tiermodelle. Tierexperimentelle Untersuchungen sind seit langem unerlässlicher Bestandteil der Erforschung neuronaler Substrate von Angst und Furcht bzw. deren pathologischer Ausprägung sowie der Neuentwicklung kausaler Behandlungsmöglichkeiten (10, 20, 21). Trotz fortschreitender technischer Möglichkeiten der Untersuchungsmethoden, z.B. in der Bildgebung, entziehen sich vor allem neurobiologische Grundlagen der direkten Untersuchung am Patienten. Darüber hinaus verbieten ethische Prinzipien die invasive Erforschung zentralnervaler Vorgänge oder die Charakterisierung neuer therapeutischer Ansätze am Menschen. Ob- gleich zahlreiche Untersuchungen auch an Zell- oder Organkulturen durchgeführt werden können, gestattet die Komplexität des Gesamtorganismus bzw. des Gehirns nur selten die Ableitung von Gesetzmäßigkeiten aus den Eigenschaften isolierter, deafferentierter Zellen. Einen experimentellen Zugriff zum Substrat der Generierung von Emotionen und Verhalten, d.h. dem Gehirn, bietet letztlich nur ein geeignetes Tiermodell. Die adäquate Nutzung von Tiermodellen ist daher in der biomedizinischen Forschung von grundlegender Bedeutung (23).

Der Terminus „Tiermodell“ wird in der psychiatrischen Grundlagenforschung als Überbegriff für verschiedene methodische Ansätze verwendet (24). Er umfasst zum einen Verhaltenstests, bei denen bestimmte Verhaltensweisen wie Angst oder Passivität durch einen situativen Kontext oder pharmakologische Manipulationen hervorgerufen werden. Zum anderen beinhaltet er Modelle, 


\section{Abb. 1 Elevated plus maze-Test}

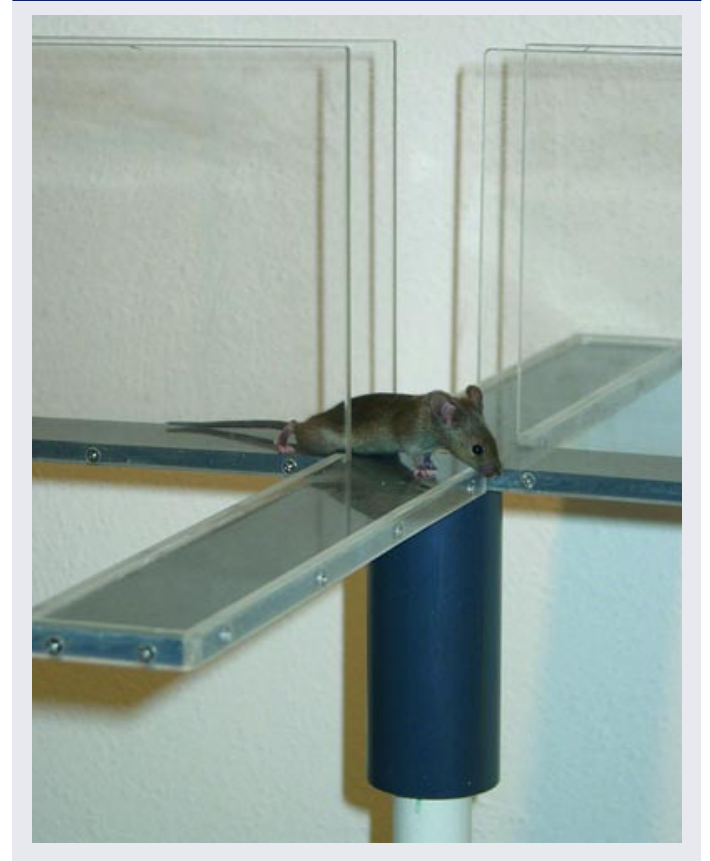

Dieser Test, der ursprünglich zur Untersuchung von anxiolytischen bzw. anxiogenen Eigenschaften von Pharmaka entwickelt wurde, beruht auf dem inneren Konflikt der Versuchstiere zwischen ihrem Explorationsdrang auf der einen und der angeborenen Aversion gegenüber offenen, hellen und erhöhten Kompartimenten auf der anderen Seite. Er gilt als einer der nicht nur ethologisch, sondern auch pharmakologisch am besten validierten experimentellen Prozeduren zur Charakterisierung des angeborenen, nicht konditionierten angstbezogenen Verhaltens. Während der fünfminütigen Expositionszeit erfolgt die Erfassung folgender Parameter: 1. Anzahl der Eintritte in die geschlossenen und offenen Arme, 2. die jeweils darin verbrachte Zeit und 3. die Latenz bis zum ersten Eintritt in einen offenen Arm. Je mehr Zeit in den offenen Armen verbracht wird, desto weniger ängstlich ist das Tier.

in welchen Tiere aufgrund von Genmanipulationen oder selektiver Zucht ein basal verändertes, angeborenes Verhalten aufweisen. Da psychiatrische Erkrankungen, neben Schizophrenie insbesondere auch affektive Störungen und Angsterkrankungen, in hohem Maße genetisch determiniert sind, bietet der letztgenannte Ansatz möglicherweise den derzeit vielversprechenderen Ansatzpunkt. Mittels selektiver Züchtung kann die genetische Basis eines Tierstammes derart verschoben werden, dass Individuen resultieren, die Verhaltensextrema aufweisen. Bei diesen können die den Verhaltensauffälligkeiten zugrunde liegenden neurobiologischen Charakteristika, wie z.B. neuroendokrine Veränderungen, untersucht werden. Vorteil ist hierbei, dass Verhaltensextreme ohne externe Manipulation im Sinne von Genmanipulation oder vorheriger Stressexposition erzielt werden, d.h. kompensatorische Mechanismen, welche die zu untersuchenden neurobiologischen Korrelate möglicherweise verfälschen könnten, sind nicht zu erwarten (21).

Grundvoraussetzung jedweder biomedizinisch-tierexperimenteller Forschung ist die Anwendung von Modellen mit phänotypischer, prädiktiver und Konstrukt-Validität (5). Dies bedeutet im Kontext psychiatrischer Erkrankungen, dass untersuchte Verhaltensweisen bzw. Emotionen evolutiv konserviert sein müssen, d.h. Tiermodell und Mensch sollten in entsprechenden Situationen auf der Verhaltensebene analog reagieren (= phänotypische Validität). Für relevante neurobiologische Mechanismen wie z.B. Neurotransmitter- und Neuromodulatorsysteme, Rezeptoren sowie Neuroanatomie von Hirnregionen kann hierbei sogar Homologie angenommen werden (= KonstruktValidität). Dies gilt insbesondere auch für Nagetiere, welche die in der biomedizinischen Forschung am häufigsten verwendeten Versuchstiere repräsentieren. Darüber hinaus sollte ein geeignetes Tiermodell das Kriterium der prädiktiven Validität erfüllen, d.h. auf Pharmaka sollte eine vergleichbare Reaktion erfolgen, welche Rückschlüsse auf eine mögliche klinische Wirksamkeit erlaubt (z.B. Anxiolyse). $\mathrm{Zu}$ beachten ist hierbei, dass sich die potentielle klinische Wirksamkeit einer therapeutischen Intervention eventuell nur in einem pathologisch veränderten System abbilden lässt. Die sorgfältige Auswahl des geeigneten Tiermodells ist somit im Kontext der prädiktiven Validität vor allem auch bei der Entwicklung neuer Therapiestrategien von grundlegender Bedeutung, da andernfalls das Risiko falsch negativer Befunde unverhältnismäßig hoch sein kann.
Eine erfolgreiche Strategie in der Untersuchung von Genen und Proteinen, die der Neurobiologie psychiatrischer Erkrankungen möglicherweise zugrunde liegen, beruht auf dem Einsatz genetisch veränderter Nager (6). Als weitere genetische Ansätze in der präklinisch-psychiatrischen Forschung seien die selektive Züchtung von Tieren sowie der Einsatz von Inzuchtstämmen, wie zum Beispiel C57BL/6- und DBA/2-Inzuchtmausstämme, genannt (25). Von großer Bedeutung unter den psychiatrischen Tiermodellen sind darüber hinaus Verhaltenstests, bei denen bestimmte Verhaltensweisen wie Angst oder Passivität durch furchtauslösende oder stressassoziierte Stimuli sowie durch gezielte pharmakologische Manipulation hervorgerufen werden (26). Als Beispiel für die am Max-Planck-Institut für Psychiatrie verwendeten ethologischen Modelle seien der Elevated plus maze- (Abb. 1), der Modified hole board- (Abb. 2) sowie der Forced swimming Test (2) genannt.

\section{Stresshormonsystem \\ (Hypothalamus-Hypo- \\ physen-Nebennieren-Achse)}

Die Reaktion auf eine akute Stresssituation ist durch zwei physiologische Mechanismen geprägt: Die erste, unmittelbare Antwort wird durch das katecholaminerge System in einer sog. "fight or flight"-Reaktion vermittelt. Die darauf folgende Stressreaktion geschieht über das Hypothalamus-Hypophysen-Nebennierenrinden (HPA)-System, das über eine adrenokortikale Ausschüttung von Kortikosteroiden - Kortisol beim Menschen, Kortikosteron bei Ratten und Mäusen - entscheidend zu einer Wiederherstellung der physiologischen Homöostase beiträgt (17). Eine Störung in den Rückkoppelungsmechanismen des Stresshormonsystems sowie durch schweren, langandauernden Stress gekennzeichnete Situationen führen $\mathrm{zu}$ chronisch erhöhten Konzentrationen an zirkulierenden Kortikosteroiden. Diese Störung im HPA-System wiederum gilt als ein wesentlicher Vulnerabilitätsfaktor in der Entstehung von chronischen Krankheiten, unter anderem von Depression und Angsterkrankungen (12). 
Auffälligkeiten in der Regulation des HPA-Systems führten, gestützt durch klinisch-neuroendokrinologische Funktionsanalysen sowie tierexperimentelle Untersuchungen, zur Formulierung der Kortikosteroidrezeptor-Hypothese der Depression (11). So nimmt man eine Minderfunktion der Glukokortikoid (GR)- und der Mineralokortikoid (MR)-Rezeptoren an, welche die HPA-Achse im Wesentlichen regulieren. Dies wiederum führt zu einer Überaktivität des Corticotropin-Releasing-Hormon (CRH)- sowie des Arginin-Vasopressin (AVP)-Systems $(13,14,16)$, die unter anderem für die Symptomatik affektiver Erkrankungen verantwortlich sind.

In diesem Zusammenhang ist von Bedeutung, dass alle bislang untersuchten Antidepressiva, welche eine hohe therapeutische Wirksamkeit bei Angsterkrankungen aufweisen, zu einer im Detail noch unbekannten Normalisierung der krankheitsassoziierten Hyperaktivität des HPA-Systems und damit der klinischen Symptomatik führen. Da dieser Effekt jedoch erst mit einer großen Latenz eintritt, wurde vorgeschlagen, im Sinne kausaler Therapiestrategien das dysregulierte CRH- bzw. AVP-System direkt pharmakologisch zu antagonisieren. Dies führte zur Entwicklung von Antagonisten des CRH-1Rezeptors (15) bzw. des AVP-1B-Rezeptors (7), welche jedoch noch nicht im klinischen Einsatz sind. Insbesondere vor diesem Hintergrund erscheint die weiterführende Charakterisierung der HPA-Achsenregulation am Menschen sowie im Tiermodell von großer Wichtigkeit, um neue, zielgerichtete Therapiemöglichkeiten für Depression und Angsterkrankungen zu ermöglichen.

\section{Humangenetische Untersuchungen bei Angsterkrankungen}

Die Untersuchung molekularbiologischer Grundlagen der Angst stellt eine immense Herausforderung dar. Genetische Analysen komplexer Verhaltensweisen werden durch die Tatsache kompliziert, dass eine Vielzahl an unterschiedlichen, interagierenden Genen involviert ist, die wiederum in steter Wechselwir- kung mit Umweltfaktoren stehen. Neben den bereits erwähnten genetischen Mausmodellen (z.B. selektives Züchten, Inzuchtmausstämme, transgene Tiere) zur Erforschung der Ätiologie psychiatrischer Erkrankungen gibt es mittlerweile zahlreiche humangenetische Untersuchungen, die eindeutig belegen, dass Angst oder Depression wesentlich durch genetische Faktoren determiniert werden (4). So schätzt man, ausgehend von Befunden von Zwillings- und Adoptionsstudien, den Einfluss genetischer Effekte auf die Pathogenese von Angsterkrankungen auf bis zu 65\% (9). Desweiteren deutet der Umstand, dass erstgradig Verwandte von Patienten mit Angsterkrankungen ein vier bis sechsfach erhöhtes Krankheitsrisiko tragen, auf einen familiären bzw. genetischen Hintergrund hin.

Die genetische Komponente dieser Erkrankung ist jedoch komplex und polygenisch, d.h. zahlreiche Gene mit kleinsten Effekten verursachen eine erhöhte Krankheitssuszeptibilität, wobei schließlich Gen-UmweltInteraktionen den Ausbruch fördern bzw. den Grad der Ausprägung determinieren können. Aufgrund dieser Komplexität konnten bisher auch nur wenige erfolgreiche genetische Analysen durchgeführt werden, die auf klassischen Kopplungsverfahren (Linkage) beruhen. Die wenigen Befunde aus humanen genomweiten Linkage-Analysen, die chromosomale Regionen verantwortlich für Angst bzw. Angsterkrankungen identifizierten, ergaben Hinweise auf krankheitsrelevante Loci auf Chromosom $7 \mathrm{p}$ (19) und 9q (27).

Linkage-Verfahren sind jedoch wenig geeignet, die Interaktionen von multiplen genetischen Loci zu verstehen. Daher wurde, gestützt durch die Entschlüsselung des humanen Genoms sowie die Etablierung von Hochdurchsatz-Genotypisierungsverfahren, eine alternative Strategie und Methode des genetischen Screenings entwickelt, die darauf beruht, genetische Polymorphismen, sog. Single nucleotide polymorphisms (SNPs), im Genom zu untersuchen (1). Ein SNP besteht aus einem einfachen Basenaustausch, der ungefähr alle 1000 Basen entwe-

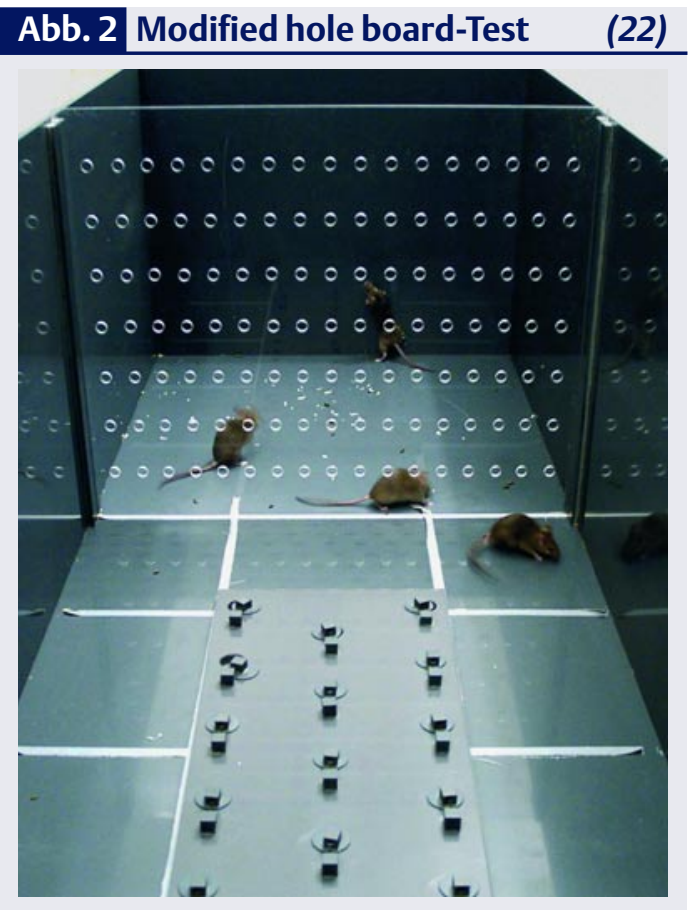

Dieser Test verbindet die Eigenschaften verschiedener selektiver Verhaltenstests und erlaubt daher die gleichzeitige Messung einer breiten Palette von Verhaltensparametern. Zusätzlich ermöglicht er die Aufrechterhaltung des Kontaktes der jeweils getesteten Tiere mit der sozialen Gruppe und verhindert so Isolationsstress während des Tests. Ängstliche Tiere zeigen u.a. ein ausgeprägtes Vermeidungsverhalten gegenüber dem ungeschützten Areal.

der in kodierenden oder nicht kodierenden Abschnitten der DNA auftritt. Zum einen können diese Polymorphismen funktionell sein, d.h. Änderungen im Transkriptom hervorrufen, zum anderen gelten sie als genetische Marker für andere krankheitsassoziierte genetische Mutationen, mit denen sie in einem Kopplungsungleichgewicht stehen (1). Aufbauend auf diese Methode werden FallKontroll-Assoziationsstudien durchgeführt. Diese unter anderem am Max-Planck-Institut für Psychiatrie in München etablierten Untersuchungen dienen dazu, genetische Assoziationen von psychiatrischen Erkrankungen, wie beispielsweise Angststörungen, mit Polymorphismen (SNPs) in spezifischen Kandidatengenen aufzuklären. Auf diese Weise konnten z.B. genetische Veränderungen im Adenosin 2a (A2aAR)-Rezeptor (3) bzw. im SerotoninTransporter (SERT) (18) gefunden werden, die signifikant häufiger bei 
Patienten mit Angsterkrankungen auftreten. Diese Marker könnten somit als Vulnerabilitätsfaktoren angesehen werden, wobei deren pathobiologische Relevanz durch weiterführende klinische und präklinische Studien im Detail noch geklärt werden muss.

Aktuelle Konzepte beruhen daher kurz zusammengefasst im Wesentlichen auf zwei Überlegungen: Erstens sollen in geeigneten Tiermodellen jene neurobiologischen Systeme, die spezifisch für angstassoziiertes Verhalten sind, identifiziert, charakterisiert und validiert werden. In einem zweiten Schritt sollen im Sinne des „translational research“ die Ergebnisse aus den präklinischen Untersuchungen an Angstpatienten mittels humangenetischer Analysen weiter validiert werden, um so möglicherweise neue, kausale Therapieansätze definieren zu können.

\section{Anxiety disorders - animal models and human genetics \\ Anxiety disorders are amongst the} most common psychiatric diseases. A causal therapy of anxiety disorders can only be attained on the basis of a more profound understanding of the neurobiology underlying anxiety in animal models and a careful extrapolation to the human situation. Today's task is to identify neurobiological systems beyond the usual suspects (such as monoamines) which are causally involved in the aetiology and pathogenesis of pathological anxiety. A multitude of genes, interacting with each other as well as with numerous environmental factors, contribute to complex behaviours and their pathological variants. To be able to draw conclusions about the human situation, the use of valid animal models is indispensable in preclinical research a fact that has long been overlooked.

\section{Key Words}

genetic - anxiety disorders polymorphism - development of psychotropic drugs

\section{Literatur}

1. Brookes AJ. The essence of SNPs. Gene 1999; 234: 177-86

2. Cryan JF, Markou A, Lucki I. Assessing antidepressant activity in rodents: recent developments and future needs. Trends in Pharmacological Sciences 2002; 23: 238-45 3. Deckert J, Nothen MM, Franke P et al. Systematic mutation screening and association study of the $A(1)$ and $A(2 a)$ adenosine receptor genes in panic disorder suggest a contribution of the $A(2 a)$ gene to the development of disease. Molecular Psychiatry 1998; 3: 81-5

4. Finn DA, Rutledge-Gorman MT, Crabbe IC. Genetic animal models of anxiety. Neurogenetics 2003; 4: 109-35

5. Geyer MA, Markou A. The role of preclinical models in the development of psychotropic drugs. In Davis KL, Charney DS, Coyle JT et al. (eds.). Neuropsychopharmacology. The Fifth Generation of Progress. Philadelphia, Baltimore, New York: Lippincott, Williams \& Wilkins, 2002: 445-56

6. Gordon JA, Hen R. Genetic approaches to the study of anxiety. Annual Review of Neuroscience 2004; 27: 193-222

7. Griebel $\mathrm{G}$ et al. The vasopressin V1b receptor as a therapeutic target in stress-related disorders. Current Drug Targets - CNS \& Neurological Disorders 2003; 2: 191-200

8. Hettema JM, Neale MC, Kendler KS. A review and meta-analysis of the genetic epidemiology of anxiety disorders. American Journal of Psychiatry 2001; 158: 1568-78

9. Hettema JM, Neale MC, Kendler KS. A review and meta-analysis of the genetic epidemiology of anxiety disorders. American Journal of Psychiatry 2001; 158: 1568-78 10. Holsboer F. Animal models of mood disorders. In Charney DS, Nestler EJ, Bunney BS (eds.). Neurobiology of mental illness. New York, Oxford: Oxford University Press, 1999: 317-32

11. Holsboer F. The corticosteroid receptor hypothesis of depression. Neuropsychopharmacology 2000; 23: 477-501

12. Holsboer $F$. The role of peptides in treatment of psychiatric disorders. Journal of Neural Transmission 2003; 64: 17-34

13. Keck ME, Holsboer F, Müller MB. Mouse mutants for the study of corticotropin-releasing hormone receptor function: development of novel treatment strategies for mood disorders. Annals of the New York Academy of Sciences 2004; 1018: 445-57

14. Keck ME et al. Reduction of hypothalamic vasopressinergic hyperdrive contributes to clinically relevant behavioral and neuroendocrine effects of chronic paroxetine treatment in a psychopathological rat model. Neuropsychopharmacology 2003; 28: 235-43

15. Keck ME et al. The anxiolytic effect of the corticotropin-releasing hormone 1 receptor antagonist R121919 depends on innate emotionality in rats. European Journal of Neuroscience 2001; 13: 373-80.

16. Keck ME et al. Vasopressin mediates the response of the combined dexamethasone/CRH test in hyper-anxious rats: Implications for pathogenesis of affective disorders. Neuropsychopharmacology 2002; 26 : 94-105

17. Korte SM. Corticosteroids in relation to fear, anxiety and psychopathology. Neuroscience and Biobehavioral Reviews 2001; 25: 117-42
18. Lesch KP et al. Association of anxiety-related traits with a polymorphism in the serotonin transporter gene regulatory region. Science 1996; 274: 1527-31

19. Logue MW et al. Bayesian analysis of a previously published genome screen for panic disorder reveals new and compelling evidence for linkage to chromosome 7. American Journal of Medical Genetics 2003; 121B: 95-9

20. Müller MB, Holsboer F, Keck ME. Genetic modification of corticosteroid receptor signalling: Novel insights into pathophysiology and treatment strategies of human affective disorders. Neuropeptides 2002; 36 : 117-31

21. Müller MB, Keck ME. Genetically engineered mice for studies of stress-related clinical conditions. Journal of Psychiatric Research 2002; 36: 53-76

22. Ohl F, Holsboer F, Landgraf R. The modified hole board as a differential screen for behavior in rodents. Behavior Research Methods, Instruments, \& Computers 2001; 33 : 392-7

23. OhI F, Keck ME. Behavioural screening in ENU-mutant mice- in search for novel animal models of psychiatric disorders. European Journal of Pharmacology 2003; 480: 219-28

24. Ohl F, Landgraf R. Animal models of anxiety. Neuroforum 2000; 4: 257-62

25. Ohl F et al. Differential analysis of behavior and diazepam-induced alterations in $\mathrm{C} 57 \mathrm{BL} / 6 \mathrm{~N}$ and $\mathrm{BALB} / \mathrm{C}$ mice using the modified hole board test. Journal of Psychiatric Research 2001; 35: 147-54

26. Shekhar A et al. Summary of a National Institute of Mental Health workshop: developing animal models of anxiety disorders. Psychopharmacology 2001; 157: 327-39

27. Thorgeirsson TE, Oskarsson H, Desnica $\mathrm{N}$ et al. Anxiety with panic disorder linked to chromosome9q in Island. American Journal of Human Genetics 2003; 72: 1221-30

\section{Korrespondenzadresse:}

Priv.-Doz. Dr. Martin E. Keck, M.Sc. Max-Planck-Institut für Psychiatrie Kraepelinstr. 2-10

80804 München

keck@mpipsykl.mpg.de 